\title{
in vitro and in vivo Evaluation of Methotrexate-Loaded Hydrogel Nanoparticles Intended to Treat Primary CNS Lymphoma via Intranasal Administration
}

\author{
Leila Pourtalebi Jahromi ${ }^{1}$, Soliman Mohammadi-Samani ${ }^{1,2}$, Reza Heidari ${ }^{1}$, Amir Azadi ${ }^{1,2}$ \\ ${ }^{1}$ Pharmaceutical Sciences Research Center, Shiraz University of Medical Sciences, Shiraz, Iran, and ${ }^{2}$ Department of \\ Pharmaceutics, School of pharmacy, Shiraz University of Medical Sciences, Shiraz, Iran
}

Received, May 23, 2018; Revised, June 20, 2018; Accepted June 28, 2018; Published, June 30, 2018.

\begin{abstract}
Purpose: Although it passes through blood-brain barrier (BBB) very poorly, methotrexate (MTX) is an important therapeutic in the treatment of many central nervous system malignancies. Accordingly, intranasal (IN) administration accompanied with a muco-adhesive chitosan-based nanoformulation is expected to overcome this problem. Methods: Nanogel containing MTX was prepared through an ionic gelation method and then characterized in terms of particle size, morphology, zeta potential, drug loading and drug release behavior. The drug release results were fitted on eight mathematical models to choose the model best describing the phenomenon. Then the nano-formulation and free drug solution in deionized water as control were administered in the nasal cavity for rats and after 15, 30, 60 and 240 minutes their brain and plasma were analyzed for MTX quantity. Results: The nano-formulation demonstrated an average particle size near $100 \mathrm{~nm}$ with a zeta potential of $18.65 \pm 1.77 \mathrm{mv}$. Loading efficiency and loading capacity were calculated to be $65.46 \pm 7.66$ and $3.02 \pm 0.34$ respectively. The Weibull model was found to be best describing the release phenomenon as a combination of swelling and Fickian diffusion. Moreover in in vivo studies, drug targeting efficiency and direct transport percentage for nanogel (test) and free drug solution (control) were $424.88 \%$ and $76.46 \%$ and $34842.15 \%$ and 99.71\% respectively. Conclusion: According to in vivo studies, nanogel produced significantly higher concentration of MTX in the brain but not in the plasma when compared to the free drug solution. Besides, in comparison to intravenous administration of the same nanogel it was indicated that intranasal administration significantly increases the brain concentration of MTX.
\end{abstract}

\section{INTRODUCTION}

Brain tumors are have poor prognosis leading to burdensome morbidities and mortalities both in families and healthcare systems $(1,2)$. Primary central nervous system lymphomas, as one of the most frequent forms of primary brain tumors in adults, are estimated to affect 4.5 per 1 million persons per year and seem to slightly increase. The median age of incidence is 65 and no differences among races have been detected in frequency of incidence, while it happens 1.33 times more in men than women $(3,4)$. The most important risk factor for primary central nervous system lymphomas is immunodeficiency both acquired and congenital and its common symptoms are headaches and behavioural/personality changes (4-6). The management of primary central nervous system lymphomas has been improved during the past decades and increased patients' quality of life. High dose intravenous (IV) methotrexate (MTX) is the backbone of treatment and whole-brain radiotherapy and other chemotherapeutics in combination with MTX has produced better clinical outcomes; although dose and frequency of chemotherapy and radiotherapy has not been optimized yet $(3,4)$.

MTX, acting as folic acid antimetabolite is a small molecule with hydrophilic nature, widely utilized in various antineoplastic regimens including those for treatment of primary central nervous system lymphomas $(7,8)$.

Although administration of MTX has been a breakthrough increasing patients survival, its poor passage through blood-brain barrier (BBB) resulting in high-dose regimens, has been a serious obstacle for the clinical practice (9).

Corresponding Author: Amir Azadi, Pharm. D., PhD; Department of Pharmaceutics, School of Pharmacy, Shiraz University of Medical Sciences, Shiraz, Iran; E mail address: aazadi@sums.ac.ir 
To put the issue into perspective, a $2^{\text {nd }}$ phase clinical trial, reporting successful implementation of $8 \mathrm{~g} / \mathrm{m}^{2}$ MTX intravenously in monotherapy, for maximum eight dose- repetitions by two-week intervals is remarkable (10). Beside the poor BBB passage, high dose MTX causes severe nephropathy because of MTX and its metabolite precipitation in renal tubules that could be life-threatening itself (5). Also hepatotoxicity and haematological adverse effects are remarkable with high dose MTX (11).

To overcome afore-mentioned problems and enhance CNS concentration of MTX in low dose, current study tries to take advantages of a mucoadhesive chitosan- based nanogel formulation accompanied with a blood-independent route of administration that is intranasal route.

Drug delivery to CNS is harshly restricted via physiological mechanisms including $\mathrm{BBB}$ and blood-cerebrospinal fluid barrier (BCSFB), as well as biochemical barriers consist of various enzymes and transporters. CNS malignancies, despite their low prevalence, have very high mortality rate that could be due to drugs' poor access to CNS (12).

Currently, conventional medicine exploits intranasal drug administration for non-systemic purposes like nasal congestion, hypersensitivity or respiratory tract infections. However, recent academic attention has been drawn to the intranasal drug administration for brain drug delivery purposes. This could be attributed to its many benefits such as being non-invasive, blood and gastrointestinal absorption independent, bypassing of BBB and hepatic first pass effect which ultimately lead to a reduced dose of drug, less systemic side effects, and increased patient compliance (13-15). Three pathways have been mentioned in the literature for nose to brain transport including olfactory nerve ending at olfactory bulb, trigeminal nerve first and second branches ending at brain stem, and absorption into systemic circulation via highly vascularized nasal epithelium. Olfactory nerve is considered to be the most important as well as the most rapid passage. Olfactory sensory neuron regenerates every 3-4 weeks in the olfactory region and during this regeneration, transport inhibitory mechanisms like tight junction proteins, enzymes and efflux transporters act poorly, forming a leaky barrier that can transit molecules via two mechanisms: first, molecules could diffuse between neurons and move through bulk transport in the interstitial fluid; second, molecules could transit transcellularly with either passive diffusion, carrier or receptor mediated transport or even endocytosis. Transcellular transit takes more time than bulk transport. However, molecules diffuse in the whole brain by cerebrospinal fluid flow as soon as they arrive at olfactory bulb. The other transport passage from nose to brain is the trigeminal nerve branches. Transport mechanism through these nerves is similar to those for olfactory nerve, although this passage from nose to brain stem is longer in distance and takes more time. The last transport passage from nose to brain, which is an indirect route, is absorption to blood stream that is closely related to molecules' lipophilicity and the ability to enter the brain through the BBB $(13,14,16)$.

Selecting a proper formulation for nose to brain drug delivery could be a key factor, affecting drug absorption from nasal epithelium. Nasal drops are not suitable because it is not possible to control dropsize leading to an unwanted dose variation. Besides, drops could easily fall out of the nasal cavity. Solid dosage forms like powders are also improper since they cause dryness and irritation. Gel-based formulations seem to be the most suitable forms because of their consistency they tend to remain in the nasal cavity for an adequate period of time while they do not irritate the mucosa $(17,18)$.

By definition, polymeric nanoparticles are nanosized $(10-1000 \mathrm{~nm})$ polymeric particles that can solve or entrap drug molecules in their matrices or encapsulate them as well as attaching drug molecules on their surface. On the other hand, hydrogels are 3D polymeric structures, distinguished by their ability of absorbing significant amounts of water. Hence, hydrogel nanoparticles or nanogels are polymeric nanoparticles with merits of both nano-sized systems and hydrogels. Nanogels' unique ability to absorb water leads to physicochemical characteristics analogous to those of living tissues more than any other group of biomaterials, including high water content, soft and rubbery consistency and low interfacial tension with water and biological fluids. Because of cross-linked structure, hydrogels tend to swell rather than being dissolved in aqueous environments and this is the key factor in determining their drug release properties (19-21).

Chitosan is a derivative of chitin, the second abundant polymer of the nature, with random order of monomers, glucosamine and N-acetyl glucosamine along the linear chain. Chitosan's appealing characteristics including biocompatibility, 
biodegradability, biosafety, possibility of various modifications (both chemically and electrostatically) and forming muco-adhesive hydrogels as well as its antitumor effects have made it a good candidate for drug delivery in the context of cancer research (19, $22,23)$. A convenient and very well established method to prepare a drug delivery system from chitosan is to insert the drug within chitosan nanoparticles, fabricated via an ionic gelation procedure. Adding solution of a polyanion (for instance tripolyphosphate) in a dropwise manner to the acidic solution of polycationic chitosan while stirring the medium provides chitosan hydrogel nanoparticles through electrostatic cross linkage with results in uni-distributed size and positive surface charge $(24,25)$.

This study was designed and conducted based on previous studies including comparison of brain delivery efficiency for MTX simple solution following administration via systemic and intranasal routes as well as declaring efficiency of nanogels for drug delivery to brain via ivroute. The Novel goal of the present study is to evaluate efficiency of the developed muco-adhesive nano-formulation in brain delivery of MTX via intranasal (in) route compared to the solution of the free drug that is available in the market. Moreover, data from our previous research including plasma and brain concentration of MTX following $i v$ administration of the same developed formulation with the same dose would be compared to the data of IN administration.

\section{MATERIALS AND METHODS}

\section{MATERIALS}

Chitosan, a low molecular weight product with an acetylation degree of $95 \%$ and viscosity of $6 \mathrm{mPa}$ in $1 \% \mathrm{w} / \mathrm{v}$ solution, was prepared from ChitoClear (Primex, Siglufjordur, Iceland). Also methotrexate (injection, USP, $1 \mathrm{~g} / 10 \mathrm{~mL}$, Mylan, France), pentasodium triphosphate (TPP; Merck, Darmstadt, Germany), and all other chemicals and solvents required for the study were purchased locally.

\section{Animals}

The study has utilized male Sprague-Dawley rats (The Animal House, Shiraz University of Medical Sciences, Shiraz, Iran) weighing between 250 and $300 \mathrm{~g}$. They were kept in standard cages with free access to water and standard rat foods.

\section{Ethics Approval for Animal Studies}

The animals were maintained depending on ethical codes and guidelines of Shiraz University of Medical Sciences as well as The Code of Ethics of the World Medical Association (Declaration of Helsinki) EC Directive 86/609/EEC addressing animal experiments. Ethics Committee of Shiraz University of Medical Sciences approved the protocols prior the experiment had been conducted (ec-p-9381-8111).

\section{Preparation of methotrexate loaded hydrogel nanoparticles}

Methotrexate loaded nanogel was prepared through an ionic gelation method involving addition of TPP solution $(1.5 \% \mathrm{w} / \mathrm{v})$ containing methotrexate to chitosan solution $(0.18 \% \mathrm{w} / \mathrm{v})$ in acetate buffer $(\mathrm{pH}=4)$ with a $7: 1$ ratio in a drop-wise manner $(26$, 27). The process was delineated to reach the final concentration of methotrexate equal to 0.075 $\mathrm{mg} / \mathrm{mL}$. After fabrication, nanoparticles were precipitated through centrifuge in $12000 \mathrm{~g}$ for $15 \mathrm{~min}$ and re-suspended in deionized water after discarding supernatant.

\section{in vitro Studies}

Particle size and size distribution:

Due to the important effect of particle size on its biological fate in the body, particle size was measured through both dynamic light laser diffraction particle size analyser (Malvern instrument, model Zetasizer 3000 HS, Malvern, Worcestershire, UK) and the static light facility (Shimadzu SADL-2101, Japan).

\section{Zeta potential}

Electrical surface charge of particles was also inspected using Zetasizer (3000 HS, Malvern, Worcestershire, UK)

\section{Morphology}

Particles' shape and plausible aggregation in nanogels ware studied through transmission electron microscopy (TEM; model EM 10C, Zeiss, Germany) with different magnifications.

\section{Drug loading}

Fabricated samples were centrifuged at $12000 \mathrm{~g}$ for $15 \mathrm{~min}$ and the supernatant was analysed using a previously described reversed phase high performance liquid chromatography (HPLC) method (28) to calculate the loading amount indirectly. Then, 
loading efficiency (LE) and loading capacity (LC) were calculated based on the following formulas:

$L E=\frac{\text { loaded } M T X \text { amount }}{\text { total MTX amount }} \times 100$

$L C=\frac{\text { loaded MTX amount }}{\text { loaded MTX amount }+ \text { chitosan weight }} \times 100$

Sum of chitosan weight and loaded MTX was assumed to represent the total particle weight.

\section{Drug release}

Drug release from nanogels was evaluated via dialysis method through a cellulose membrane (avg. flat width $33 \mathrm{~m}$, typical molecular weight cutoff $=14000$, SIGMA-ALDRICH) in phosphate buffered solution (PBS) medium at $\mathrm{T}=25^{\circ} \mathrm{c}$ and $\mathrm{pH}=7.4$ considering the sink condition. To determine the effect of nanogels on drug release, the process was repeated for the free drug solution. Sampling from the release medium of nanogel was done at $0.25,0.5,1,2,5,7,8$, and $24 \mathrm{~h}$ and from that of free drug solution was performed at $0.25,0.5,1,2,5,7$ and $8 \mathrm{~h}$. Then, samples were analysed by the mentioned HPLC method.

For a further kinetic study, the release data were fitted to the following 8 conventional mathematical release models. In the formulas, $f$ represents drug amount released at time of $t, K$ stands for constant of equation and $t_{d}$ introduces the delay up to release initiation. $\beta$ is also a representative of drug release graph.

Zero order:

$$
f=K . t
$$

First order:

$\ln (1-f)=-K . t$

Higuchi:

$f=K \cdot t^{\frac{1}{2}}$

Hixson-Crowell:

$$
1-(1-f)^{\frac{1}{3}}=-K \cdot t
$$

Square root of mass:

$$
1-(1-f)^{\frac{1}{2}}=-K . t
$$

Three seconds root of mass:

$$
1-(1-f)^{\frac{2}{3}}=-K . t
$$

Weibull:

$\ln [-\ln (1-f)]=\beta \cdot \ln \left(t_{d}\right)+\beta \cdot \ln (t)$

Korsmeyer-Peppas:

$\ln (f)=\ln (K)+n \cdot \ln (t)$

Then, sum square of total variation (SST), sum square of errors (SSE), sum square of regression (SSR), coefficient of determination $\left(\mathrm{R}^{2}\right)$, akaike information criteria (AIC), and absolute percent of error (E) were calculated for each equation to evaluate their accuracy and power of prediction. Further, formulations used to calculate aforementioned parameters are as follows $(29,30)$.

Sum square of total variation (SST):

$S S T=\sum_{i=1}^{n}\left(y_{i}-y\right)^{2}$

Sum Square of errors (SSE):

$S S E=\sum_{i=1}^{n} e^{2}=\sum_{i=1}^{n}\left(y_{i}-y f_{i}\right)^{2}$

Sum square of regression (SSR):

$S S R=S S T-S S E$

Coefficient of determination $\left(\mathrm{R}^{2}\right)$ :

$R^{2}=1-(S S E / S S T)$

Akaike information criteria (AIC):

$A I C=N \times \ln (S S E)+2 \times p$

Absolute percent of Error (E):

$E=\frac{100}{N} \sum_{i=1}^{N} \frac{\mid \text { fal }_{i}-\text { fobs }_{i} \mid}{f o b s_{i}}$

Number of errors (NE):

$N E_{i}=\frac{100 \times n_{i}}{N}$

\section{in vivo Studies}

Preparation of rats for drug administration:

Animals were anaesthetized using a ketaminexylazine cocktail $(70 \mathrm{mg} / \mathrm{kg}$ ketamine and $7 \mathrm{mg} / \mathrm{kg}$ xylazine). Then their trachea was intubated with feeding tube number 6 in order to enable animals to breathe (31).

\section{Drug administration}

For sampling at each time point, rats were divided into two groups of test and control, each containing five animals. For the test group, nanogel was administered nasally after preparation, separation and resuspension, to suffice a $25 \mu \mathrm{g}$ of methotrexate in $1 \mathrm{~kg}$ of body weight utilizing a $100 \mu 1$ sampler. For 
the control group, free drug solution $(200 \mu \mathrm{g} / \mathrm{mL})$ prepared by diluting the commercial vial of methotrexate in deionized water and was administered in nasal cavity with the same method and dose as the test subjects.

\section{Obtaining tissue specimens}

Five animals from both test and control groups were sacrificed at 15, 30, 60 and 240 minutes after drug administration. A blood sample was taken and the whole brain was harvested after each animal's death. Then blood samples were centrifuged at $2000 \mathrm{~g}$ for $20 \mathrm{~min}$ to eliminate cellular compartment. Thereafter, plasma and brain specimens were frozen at $-70^{\circ} \mathrm{C}$ till the quantification analyses.

\section{Quantification of methotrexate in tissue samples}

The same previously cited HPLC method was used to quantify MTX in whole brain and plasma samples (28).

\section{Calculating neuro-pharmacokinetic parameters}

Two main parameters describing neuropharmacokinetics of drugs, administered via nasal route, are drug targeting efficiency (DTE) and direct transport percentage (DTP) calculating through following formulas.

$$
\begin{aligned}
& D T E=100 \times \frac{\left(A U C_{\text {brain }} / A U C_{\text {plasma }}\right)_{i n}}{\left(A U C_{\text {brain }} / A U C_{\text {plasma }}\right)_{i v}} \\
& D T P=100 \times \frac{A U C_{\text {brain }(\text { in })}-B_{x}}{A U C_{\text {brain }(\text { in })}}
\end{aligned}
$$

Where:

$B_{x}=\frac{A U C_{\text {brain }(i v)} \times A U C_{\text {plasma }}(\text { in })}{A U C_{\text {plasma }}(\text { iv })}$

AUC in formulas above represents area under concentration-time curve while in and $i v$ indices represent intranasal and intravenous route of administration.

\section{STATISTICAL ANALYSIS}

To recognize significant differences between MTX concentration in brain and plasma samples of test and control groups, f-test was performed to control homogeneity of variances (significance level $=0.05$ ); then average of groups were compared through homoscedastic or heteroscedastic t-test (significance level=0.05).
Finally, students' t-test was administered to compare the study findings with a previously done experiment on rats (32), receiving the same dose of the same formulation through the iv route (significance level $=0.05$ ).

All of the analyses were done, utilizing StatPlus:mac LE (AnalystSoft. Inc.; 2015) and SPSS statistics 23.0 (IBM; 2015).

\section{RESULTS}

\section{in vitro Studies}

\section{Particle size and size distribution}

Both static and dynamic light LASER diffraction methods declared unimodal curves as well as normal size distribution for nanoparticles. Figure 1 represents the results. Accordingly, dynamic method Z-average is about $110.18 \pm 17.70 \mathrm{~nm}$ with a polydispersity index (PDI) of $0.26 \pm 0.07$ (Figure 1 A), while for the static method, median diameter considering number, volume and area of particles is $75.33 \pm 1.15 \mathrm{~nm}, 93.67 \pm 4.04 \mathrm{~nm}$ and $86.33 \pm 2.89 \mathrm{~nm}$, respectively (Figure $1 \mathrm{~B}$ ).
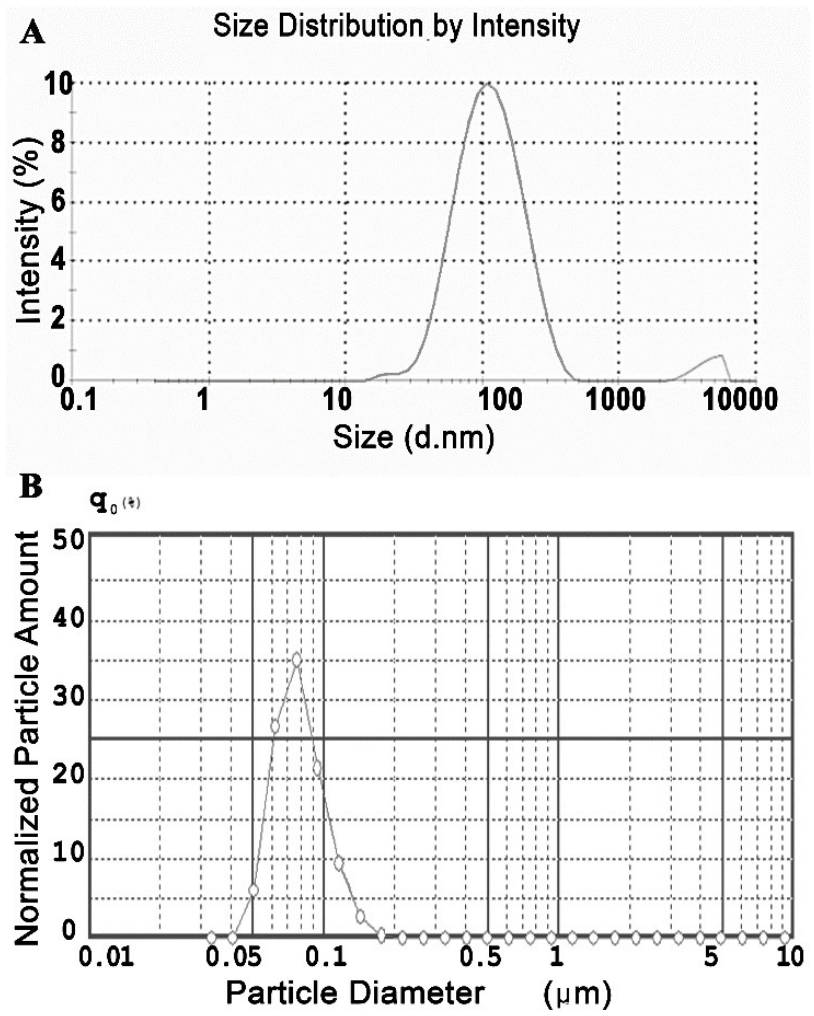

Figure 1. Dynamic light laser diffraction (A) and static light laser diffraction measured based on the number of most frequent particles (B). 


\section{Morphology}

Nanoparticles are distinguishable as quite spherical particles with smooth surface, containing small vacuoles of water. Based on the scale of TEM images, determined size and size distribution of nanoparticles are in a good agreement with the static and dynamic light Laser diffraction results (Figure 2 A).

As reported in the literature, continuous imaging of the chitosan hydrogel nanoparticles with TEM, results in absorption of energy from emitted electrons. Consequently, little vacuoles tend to aggregate and form a large vacuole, from which water evaporates and leaves the chitosan matrix (33).

\section{Drug Loading Parameters}

In the art of drug formulating, it is noteworthy that loading parameters should be high enough to deliver optimum amount of drug using the least plausible excipients. Especially, this is true for polymers based on their safety issues and intake limitations. Aforementioned method of preparation for hydrogel nanoparticles lead to a LE of $65.46 \pm 7.66 \%$ and a LC of $3.02 \pm 0.34 \%$. Appealing characteristics about the formulation are the acceptable LE and the very high safe dose of chitosan beside its pharmacological effects (26) (34).

\section{Zeta potential}

Based on an electrophoretic mobility mechanism, zeta potential of prepared nanogel determined to be $18.65 \pm 1.77 \mathrm{mv}$.

\section{Drug release}

Figure 2.B illustrates release pattern of MTX from both nanogels and the drug solution. Curve fitting analyses on 8 conventional mathematical models were applied to reach a more lucid illustration of release mechanism. Analyses showed that Weibull model best described the MTX release phenomenon from nanogels due to the highest $\mathrm{R}^{2}$, the least AIC, and error and the highest $\mathrm{NE}<10 \%$ as well as $\mathrm{NE}<20 \%$. All the curve-fitting parameters calculated for each eight mathematical release models are mentioned in Table 1.

\section{Quantification of MTX in tissue samples}

Table 2 represents MTX concentration in brain and plasma of both test and control groups at sampling points. To quantify MTX in the brain tissue more precisely, amount of MTX in approximate $0.06 \mathrm{~mL}$ blood nourishing the brain is excluded from reported values (35).
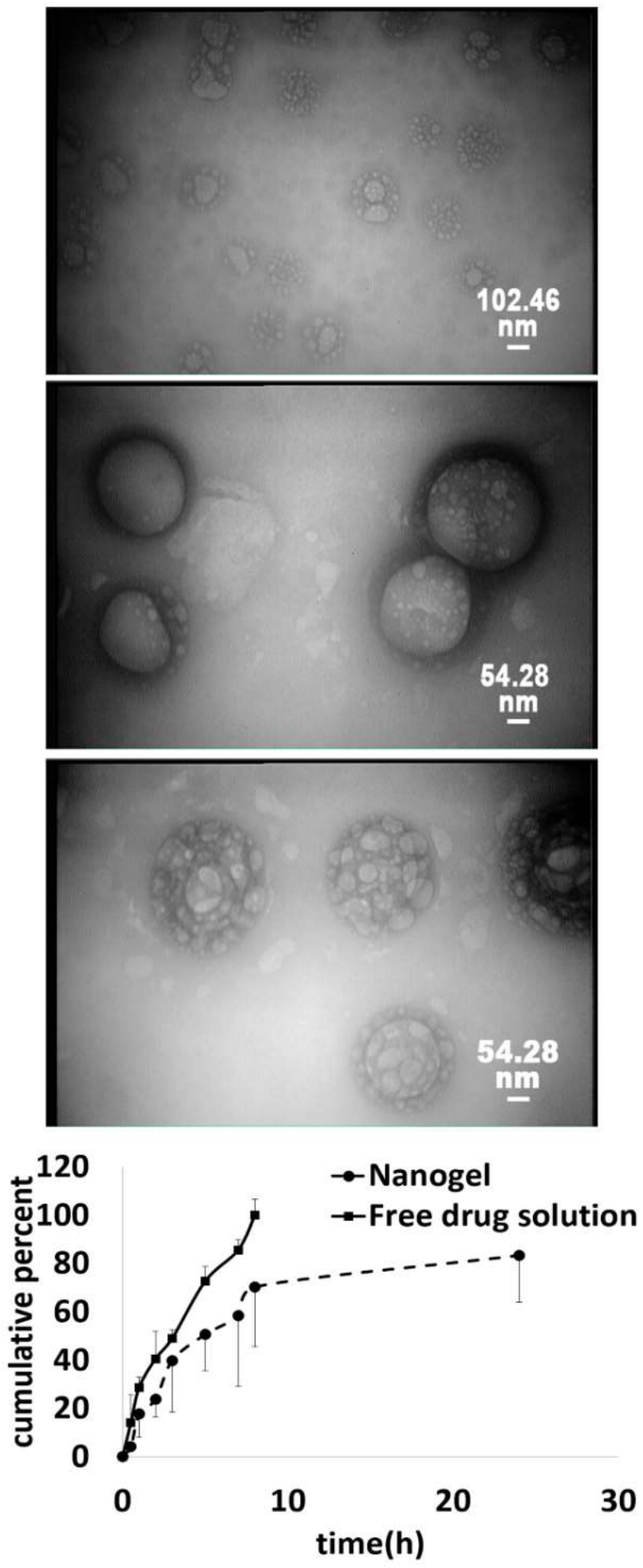

Figure 2. TEM images of nanoparticles in $9.6 \times 10^{4}, 1.8$ $\times 10^{5}$ and $1.8 \times 10^{5}$ magnitudes respectively $(\mathrm{A})$; cumulative drug release from solution and nanogel test in triplicate (B). 
J Pharm Pharm Sci (www.cspsCanada.org) 21, 305 - 317, 2018

Table 1. Calculated parameters of accuracy and power of prediction for eight mathematical release models

\begin{tabular}{|c|c|c|c|c|c|c|c|c|c|c|}
\hline \multirow[t]{2}{*}{ Model } & \multirow[t]{2}{*}{ b } & \multirow[t]{2}{*}{$\mathbf{a}$} & \multirow[t]{2}{*}{$\mathbf{R}^{2}$} & \multirow[t]{2}{*}{ SST } & \multirow[t]{2}{*}{ SSE } & \multirow[t]{2}{*}{ SSR } & \multirow[t]{2}{*}{ AIC } & \multicolumn{2}{|c|}{$\% \mathrm{NE}<$} & \multirow[t]{2}{*}{ Error\% } \\
\hline & & & & & & & & 10 & 20 & \\
\hline Zero order & 0.03 & 0.28 & 0.69 & 0.92 & 0.56 & 0.36 & 1.32 & 12.5 & 25.0 & 51.7 \\
\hline First order & -0.07 & -0.24 & 0.87 & 0.52 & 0.09 & 0.43 & -13.57 & 37.5 & 50.0 & 77.3 \\
\hline Higuchi & 0.19 & 0.02 & 0.88 & 0.52 & 0.06 & 0.46 & -16.40 & 0 & 62.5 & 45.1 \\
\hline $\begin{array}{l}\text { Hixson- } \\
\text { Crowell }\end{array}$ & 0.02 & 0.09 & 0.82 & 0.65 & 0.11 & 0.54 & -11.86 & 25.0 & 50.0 & 82.5 \\
\hline $\begin{array}{l}\text { Square root of } \\
\text { mass }\end{array}$ & 0.02 & 0.13 & 0.79 & 0.52 & 0.12 & 0.40 & -11.12 & 12.5 & 50.0 & 84.2 \\
\hline $\begin{array}{l}\text { Three-second } \\
\text { root of mass }\end{array}$ & 0.02 & 0.17 & 0.76 & 0.52 & 0.13 & 0.39 & -10.38 & 12.5 & 50.0 & 85.7 \\
\hline Weibull & 0.09 & -1.96 & 0.93 & 0.52 & 0.03 & 0.49 & -21.10 & 37.5 & 75.0 & 18.9 \\
\hline $\begin{array}{l}\text { Korsmeyer- } \\
\text { Peppas }\end{array}$ & 0.7 & -2.03 & 0.87 & 1.16 & 0.30 & 0.86 & -3.63 & 25.0 & 50.0 & 25.7 \\
\hline
\end{tabular}

Table2. MTX Concentration in biological samples of test and control groups

\begin{tabular}{|c|c|c|c|c|}
\hline \multirow[b]{2}{*}{ Time } & \multicolumn{4}{|c|}{ MTX Concentration (administered formulation) } \\
\hline & $\begin{array}{c}\text { Brain (solution), } \\
\text { (ng/g) }\end{array}$ & $\begin{array}{c}\text { Brain (nanogel,) } \\
\text { (ng/g) }\end{array}$ & $\begin{array}{c}\text { Plasma (solution) } \\
(\mathrm{ng} / \mathrm{mL})\end{array}$ & Plasma (nanogel) $(\mathrm{ng} / \mathrm{mL})$ \\
\hline 15 & $88 \pm 21$ & $1366 \pm 484$ & $59 \pm 32$ & $61 \pm 19$ \\
\hline 30 & $112 \pm 38$ & $1938 \pm 293$ & $135 \pm 36$ & $117 \pm 52$ \\
\hline 60 & $95 \pm 41$ & $2055 \pm 562$ & $118 \pm 48$ & $110 \pm 32$ \\
\hline 240 & $53 \pm 12$ & $1116 \pm 298$ & $198 \pm 117$ & $351 \pm 207$ \\
\hline
\end{tabular}

in vivo Studies

Figures $3 \mathrm{~A}$ and $\mathrm{B}$ also exhibit profile of MTX concentration in brain and plasma of test and control groups at each sampling time. Besides, figure $3 \mathrm{C}$ demonstrates how brain/plasma ratio of MTX concentration changes over time. Significant differences between means of MTX concentration for test and control groups in each sampling time are emphasized by *.

Negative slope for integration plot (Figure 3 D) restricts more detailed interpretations of drug uptake and clearance from the brain but it could be inferred that dominant MTX flow is from brain to blood, which declares that direct nose to brain transport has greater possibility than entering the brain indirectly after blood absorption.

In our previous research, the same nanogel with same dose of MTX was administered through iv route for similar animals (32). Figures $4 \mathrm{~A}$ to $4 \mathrm{D}$ make comparison between aforesaid study findings and those of the present investigation and * represents significant difference where applied.

\section{Neuropharmacokinetic evaluations}

For control group DTE\% and DTP\% were 34842.15 and 99.71, while for the test group indices were 424.88 and 76.46 , respectively.

Figures 3 A and B also exhibit profile of MTX concentration in brain and plasma of test and control groups at each sampling time. Besides, figure $3 \mathrm{C}$ demonstrates how brain/plasma ratio of MTX concentration changes over time. Significant differences between means of MTX concentration for test and control groups in each sampling time are emphasized by*. 
Negative slope for integration plot (Figure $3 \mathrm{D}$ ) restricts more detailed interpretations of drug uptake and clearance from the brain but it could be inferred that dominant MTX flow is from brain to blood, which declares that direct nose to brain transport has greater possibility than entering the brain indirectly after blood absorption.

In our previous research, the same nanogel with same dose of MTX was administered through iv route for similar animals (32). Figures $4 \mathrm{~A}$ to $4 \mathrm{D}$ make comparison between aforesaid study findings and those of the present investigation and * represents significant difference where applied.

\section{DISCUSSION}

\section{in vitro Studies}

\section{Particle size and size distribution}

Despite the great number of studies on nose to brain drug delivery during the last decade, there is no comprehensive data about how particle size affects the amount of drug entrance to the brain and no exact limitation has been reported in the literature.

Electron microscopy has revealed that olfactory axons in human have diameters about 100-700 nm and theoretically, smaller particles could transport via transcellular route through these axons (36). Accordingly, obtained particle size (almost $100 \mathrm{~nm}$ ) seems to be in a suitable range for transcellular transport within the brain.

\section{Zeta potential}

Effect of nanocarrier surface charge on brain absorption from intranasal route is also a matter of controversy. Because of the present glycocalyx (with negative charge) on the $\mathrm{BBB}$, it is expected that positively-charged particles are easily attracted while negative-charge particles are repelled. Although, there are experiments showing that both cationic and anionic nanoparticles can cross the BBB. For example, Gabal et al. investigated the effect of surface charge of cationic and anionic nanostructured lipid nanocarriers for intranasal drug delivery purpose. According to their study, cationic particles contributed to higher $\mathrm{C}_{\max }$ of drug in the brain (the difference was not statistically significant) while anionic particles indicated the highest DTE\% which was 1.2-fold higher than that of cationic carriers (37).
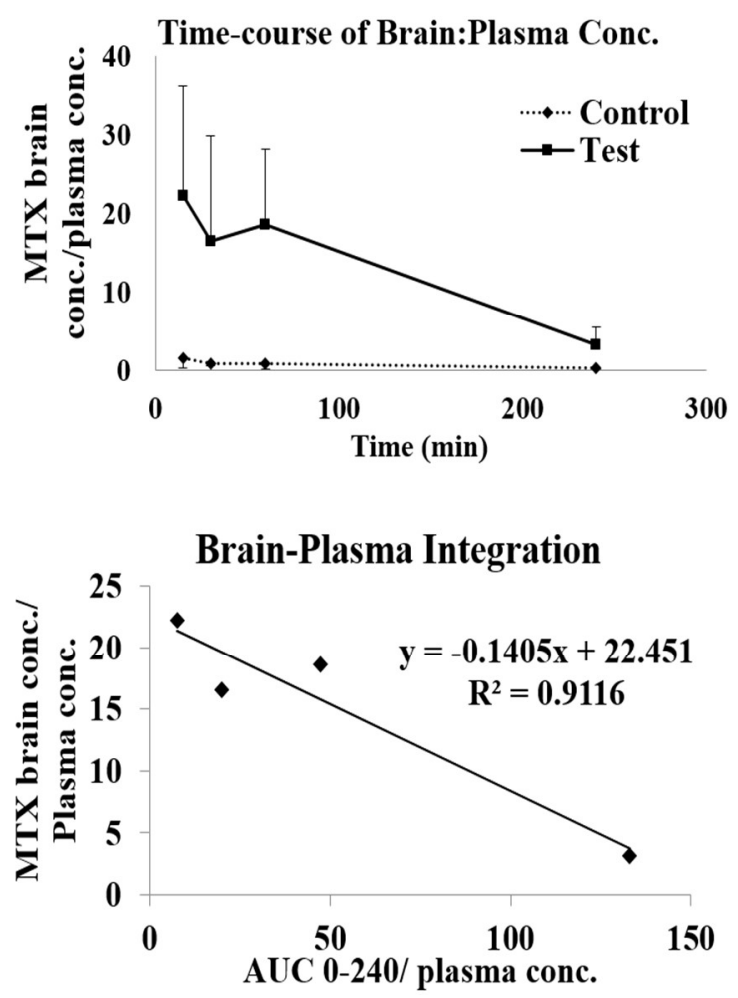

Figure 3. Methotrexate (MTX) brain and plasm concentration. following in administration of test (nanogel) and control (solution). Time-course pf changes in MTX brain:plasma of concentration ratio and relationship of such ratio with AUC in plasma are depicted. $\mathrm{n}=5$ in all of the groups; $*$ depicts $p<0.05$. 

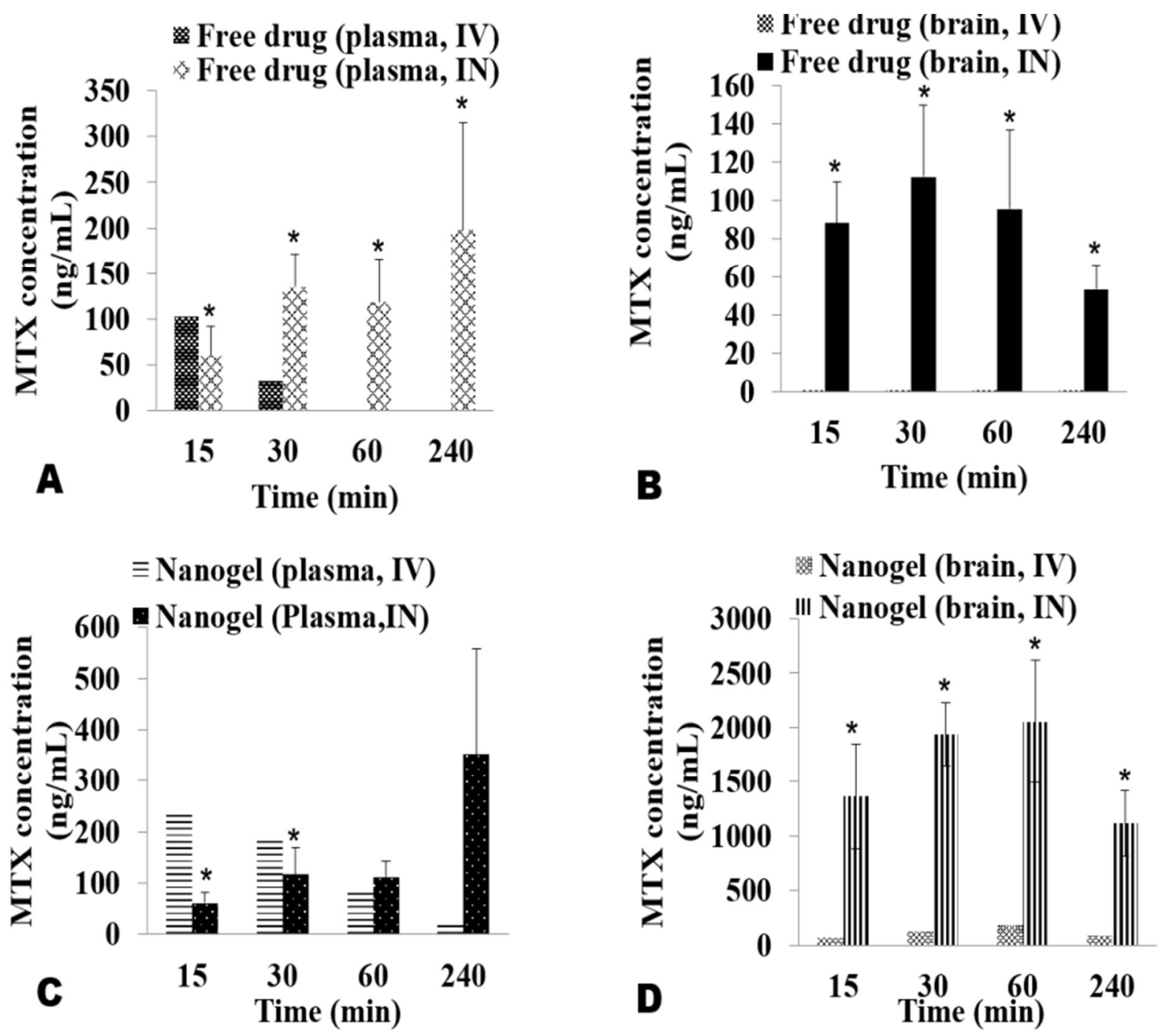

Figure 4. A. Drug conc. in plasma after in and $i v$ administration of drug solution; B, Drug conc. in brain after in and $i v$ administration of drug solution; $\mathrm{C}$, Drug conc. in plasma after in and $i v$ administration of nanogel; D, Drug conc. in brain after in and $i v$ administration of nanogel. $\mathrm{n}=5$ /group; * significantly different from $\mathrm{IV}, \mathrm{p}<0.05$ ).

\section{Morphology}

Considering the morphology of particles and the physicochemical characteristics of the polymer, MTX could be associated to the nanoparticles in several ways. MTX molecules could be entrapped within the polymeric matrix, dissolved in the entrapped water (vacuoles) or be attached to the surface of particles. Although it is proven that chitosan opens tight junctions transiently and $\mathrm{i}$ facilitates paracellular transport of polar drugs, it is not clear whether the increase in brain drug absorption (via nasal route) is because of transporting encapsulated drug or simply is a result of increased nasal retention time due to mucoadhesiveness (38).

\section{Drug release}

In the case of drug solution, MTX molecules pass through dialysis membrane and reach equilibrium with a short delay which could be considered as the time necessary for the membrane to saturate. According to the nanogels curve, release takes place in two distinguishable phases: 1)a primary rapid release, which is believed to be a result of surface absorbed MTX molecules detachment followed by the release of molecules from matrix pores and 2)a secondary slower phase, caused by drug release in conclusion of matrix erosion $(26,39)$. Comparing slope of release curve for the drug solution and nanogel also demonstrates that nanogel leads to a slight sustained release. 
Many mathematical models have been developed to describe release phenomenon from various modified release formulations. Zero order models describe systems in which the release rate is independent of remaining amount of the drug in the systems. On the other hand, first order models portray systems in which the release is always dependent on the system's drug content. Higuchi is another comprehensive model, obtained from Fickian diffusion that relates release rate to second root of time. Hixson-Crowell is another model, particularly applied to describe systems eroding over time. The model helps delineate a relationship between cube root of time and the release rate. Square root of mass and three second root of mass are also models derived from Hixson-Crowell to provide a better description of the release phenomenon for some formulations. Weibull model is an empirical model, describing both immediate and delayed release from various systems. Korsmeyer-Peppas is another semi-empirical model used for curve fitting analyses $(29,40)$, in which slope of the curve would help predict the release mechanism. Accordingly, $\mathrm{n}=0.43$ represents Fickian diffusion, $\mathrm{n}=0.85$ represents polymer swelling and between 0.43 and 0.85 the mechanism is anomalous transport that is a combination of afore-mentioned mechanisms (41). For the current data, the scale portrays release mechanism as a combination of Fickian diffusion and swelling as it is expected for hydrogel nanoparticles $(19,41,42)$.

\section{in vivo Studies}

In the present study, brain MTX concentration following in administration of both MTX solution and nanogels is significantly higher than that following iv administration (Figures 4B and 4D). Figure $3 \mathrm{~A}$ shows the MTX peak concentration at 60 min meaning the MTX brain absorption rate is higher than its elimination while the ratio of absorption and elimination reverses after 60 minutes. Furthermore, plasma MTX concentration curves reveal that after $i v$ administration, concentration abates by the time, which is expected looking at elimination procedure from central compartment; while following nasal utilization, plasma concentration rises because of direct transport from nose to blood as well as indirect transport from brain to blood. It is understood that, if sampling has been continued, decline in MTX plasma concentration would have been observed and that would happen at the point, when elimination surpasses absorption (Figure $3 \mathrm{~B}$ ). Also as figure $3 \mathrm{D}$ exhibits, for nanogel formulation brain/plasma concentration of MTX decreases over time; hence it could be concluded that nanogel affects brain absorption more than maintaining drug concentration in the brain.

Shingaki et al. (43) and Wang et al. (44) compared in and systemic administration routes for methotrexate aqueous solution. Shingaki and colleagues used 3 doses of 1.25 and $2.5 \mathrm{mg}$ MTX in the form of solution with two-day both intranasally and intraperitoneally to glioma-bearing rats and after the same period of time, tumor weights following intranasal administration were $25 \%$ of that in rats treated intraperitoneally (43). In the study by Wang, $3.2 \mathrm{mg} / \mathrm{kg}$ methotrexate was administered to rats divided into two groups, receiving drug intranasally and intravenously. Analysing biological samples in various time points demonstrated that MTX concentration in plasma following in administration was significantly lower than that of $i v$ administration, while drug concentration detected in CSF was significantly higher. Drug targeting index $\left(D T I=\left(\left(A U C_{C S F} / A U C_{\text {Plasma }}\right)_{\text {in }} /\left(A U C_{\text {CSF }} / A U C_{\text {Plasma }}\right)_{i v}\right)\right)$

for methotrexate solution was calculated to be 21.7; hence it could be concluded that MTX was transported into brain directly via the nasal route (44). Current study also supports the previous findings. The first remarkable fact about the calculated DTE parameters is their large quantity, indicating that brain MTX uptake is far higher following in administration compared to $i v$ administration for both the drug solution and the nanogel formulation at the mentioned MTX dose. Also higher DTE in case of drug solution compared to nanogel shows that in route affects absorption from drug solution more than that of nanogel. It should be noted that the difference in the quantity of DTE for drug solution in the present study and Wang's study (44), could be attributed to MTX absorption that is dose-dependent and such minute dose intravenously administered in the current study, would lead to near-zero brain concentration and produces such DTE accordingly, although the trend seems to be the same in low doses as well as high doses.

DTP is the other important pharmacokinetic parameter describing probability of indirect transport to brain following blood absorption. For the drug solution, the DTP of 99.71 states that the dominant pathway of MTX transport due to the drug solution from nose to brain is mainly direct transport. In other 
words, plausibility of MTX solution, absorbed from nose to blood to enter the brain is only $0.29 \%$, while this value for MTX-loaded nanogel is $23.45 \%$. These findings are in good agreement with integration plot interpretations (Figure $3 \mathrm{D}$ ).

Drug delivery to CNS has always been a challenge especially for hydrophilic molecules like MTX. Recently, nanotechnology has made a paradigm shift in the context of drug delivery and many research teams have made attempts to take advantages of nano-world to solve puzzles of conventional medicine. Hydrogel nanoparticles, resembling living tissues, are powerful tools in nanomedicine and chitosan as a hydrogel-forming natural polymer with appealing properties has been widely used for drug delivery to the brain in literature as well as the present study. The other strategy used to increase brain concentration of MTX is replacing $i v$ route of administration with in route. In conclusion, in the afore-mentioned dose, nanogel formulation significantly provides higher brain concentration than the free drug solution when administered nasally. Here, the important question that should be considered in further studies is whether nanogel is transported to brain or simply the rise in brain concentration is because of nanogel's muco-adhesivness providing adequate time for drug release. Looking at the DTP, it could be perceived that for MTX in both formulations, there is a higher probability that absorption takes place directly from nose to brain rather than indirect brain absorption following entrance to the systemic blood circulation. After all, in route seems to be completely superior to $i v$ route, generating higher brain concentration utilizing either free drug solution or nanogel, although further pharmacokinetic studies with more sampling time points are required.

\section{ACKNOWLEDGMENTS}

This study was part of a Pharm D thesis supported by Shiraz University of Medical Sciences (SUMS; Grant no. 93-01-36-8111). Authors also would like to appreciate Dr. Pedram Rafiei from the University of Saskatchewan for his valuable comments that improved the quality of the present work.

\section{REFERENCES}

1. Cooper I, Last D, Guez D, Sharabi S, Goldman SE, Lubitz I, et al. Combined local blood-brain barrier opening and systemic methotrexate for the treatment of brain tumors. Journal of Cerebral Blood Flow \& Metabolism. 2015;35(6):967-76. doi: 10.1038/jcbfm.2015.6.

2. De Robles P, Fiest KM, Frolkis AD, Pringsheim T, Atta C, St Germaine-Smith C, et al. The worldwide incidence and prevalence of primary brain tumors: a systematic review and meta-analysis. Neurooncology. 2015;17(6):776-83. doi: 10.1093/neuonc/nou283.

3. Ricard D, Idbaih A, Ducray F, Lahutte M, HoangXuan K, Delattre J-Y. Primary brain tumours in adults. The Lancet. 2012;379(9830):1984-96. doi: 10.1016/S0140-6736(11)61346-9.

4. Doucet S, Kumthekar P, Raizer J. Primary central nervous system lymphoma. Current treatment options in oncology. 2013;14(2):185-97. doi: 10.1007/s11864-013-0227-7.

5. Fraser E, Gruenberg K, Rubenstein JL. New approaches in primary central nervous system lymphoma. Chinese clinical oncology. 2015;4(1):11. doi: 10.3978/j.issn.2304-3865.2015.02.01.

6. Behin A, Hoang-Xuan K, Carpentier AF, Delattre JY. Primary brain tumours in adults. The Lancet. 2003;361(9354):323-31. Doi: 10.1016/S01406736(03)12328-8.

7. Sweetman SC. Methotrexate monograph. Martindale: The Complete Drug Reference. 36 ed: Pharmaceutical Press; 2009. p. 745-51.

8. Methotrexate monograph. In: Charles F. Lasy LLA, Morton P. Goldman, Leonard L. Lance., editor. Drug Information Handbook: A Comprehensive Resource for All Clinicians and Healthcare Professionals: LexiComp; 2010. p. 1056-61.

9. Gao K, Jiang X. Influence of particle size on transport of methotrexate across blood brain barrier by polysorbate 80-coated polybutylcyanoacrylate nanoparticles. Int J Pharm. 2006;310(1-2):213-9. doi: 10.1016/j.ijpharm.2005.11.040.

10. Gerstner E, Carson KA, Grossman SA, Batchelor TT. Long-term outcome in PCNSL patients treated with high dose methotrexate and deferred radiation. Neurology. 2008;70(5):401-2. doi: 10.1212/01.wnl.0000300671.37279.0e.

11. Green MR, Chowdhary S, Lombardi KM, Chalmers LM, Chamberlain M. Clinical utility and pharmacology of high-dose methotrexate in the treatment of primary CNS lymphoma. Expert Rev Neurother. 2006;6(5):635-52. doi: 10.1586/14737175.6.5.635.

12. Stein M, Kuten A, Ben-Arieh Y, Cohen Y, Haim N. [Primary brain lymphoma]. Harefuah. 1991;121(12):4-8.

13. Pardeshi CV, Belgamwar VS. Direct nose to brain drug delivery via integrated nerve pathways bypassing the blood-brain barrier: an excellent platform for brain targeting. Expert Opin Drug Deliv. 
2013;10(7):957-72.

doi: 10.1517/17425247.2013.790887.

14. Ruigrok MJ, de Lange EC. Emerging Insights for Translational Pharmacokinetic and PharmacokineticPharmacodynamic Studies: Towards Prediction of Nose-to-Brain Transport in Humans. AAPS J. 2015;17(3):493-505. doi: 10.1208/s12248-015-9724$\mathrm{x}$.

15. Vyas TK, Shahiwala A, Marathe S, Misra A. Intranasal drug delivery for brain targeting. Curr Drug Deliv. 2005;2(2):165-75.

16. Djupesland PG, Messina JC, Mahmoud RA. The nasal approach to delivering treatment for brain diseases: an anatomic, physiologic, and delivery technology overview. Ther Deliv. 2014;5(6):709-33. doi: $10.4155 /$ tde.14.41.

17. Bahadur S, Pathak K. Physicochemical and physiological considerations for efficient nose-tobrain targeting. Expert Opin Drug Deliv. 2012;9(1):19-31. $10.1517 / 17425247.2012 .636801$

18. Misra A, Kher G. Drug delivery systems from nose to brain. Curr Pharm Biotechnol. 2012;13(12):2355-79.

19. Hamidi M, Azadi A, Rafiei P. Hydrogel nanoparticles in drug delivery. Adv Drug Deliv Rev. 2008;60(15):1638-49. doi: 10.1016 / j.addr. 2008.08.002.

20. Kabanov AV, Vinogradov SV. Nanogels as pharmaceutical carriers: finite networks of infinite capabilities. Angew Chem Int Ed (English). 2009;48(30):5418-29. doi: 10.1002/anie.200900441.

21. Sivaram AJ, Rajitha P, Maya S, Jayakumar R, Sabitha M. Nanogels for delivery, imaging and therapy. Wiley Interdiscip Rev Nanomed Nanobiotechnol. 2015;7(4):509-33. doi: 10.1002/wnan.1328.

22. Hu L, Sun Y, Wu Y. Advances in chitosan-based drug delivery vehicles. Nanoscale. 2013;5(8):3103-11. doi: $10.1039 / \mathrm{c} 3 \mathrm{nr} 00338 \mathrm{~h}$.

23. Wang JJ, Zeng ZW, Xiao RZ, Xie T, Zhou GL, Zhan $\mathrm{XR}$, et al. Recent advances of chitosan nanoparticles as drug carriers. Int J Nanomedicine. 2011;6:765-74. doi: 10.2147/IJN.S17296.

24. Hamidi M, Azadi A, Mohamadi-Samani S, Rafiei P, Ashrafi H. Valproate-Loaded hydrogel nanoparticles: Preparation and characterization. J Appl Polym Sci. 2012;124(6):4686-93. doi: 10.1002/jps.22395.

25. Fernandez-Urrusuno R, Calvo $\mathrm{P}$, Remuñán-López $\mathrm{C}$, Vila-Jato JL, Alonso MJ. Enhancement of nasal absorption of insulin using chitosan nanoparticles. Pharmaceut Res. 1999;16(10):1576-81.

26. Azadi A, Hamidi M, Khoshayand M-R, Amini M, Rouini M-R. Preparation and optimization of surfacetreated methotrexate-loaded nanogels intended for brain delivery. Carbohydr Polym. 2012;90(1):46271. doi: 10.1016/j.carbpol.2012.05.066.

27. Hamidi M, Azadi A, Ashrafi H, Rafiei P, MohamadiSamani S. Taguchi orthogonal array design for the optimization of hydrogel nanoparticles for the intravenous delivery of small-molecule drugs. Journal of Applied Polymer Science. 2012;126(5):1714-24.

28. Pourtalebi Jahromi L. Mohammadi-Samani S, Ashrafi H., Azadi A. A Reversed-phase High Performance Liquid Chromatography (HPLC) method for bio-analysis of Methotrexate. Trends in pharmaceutical sciences. 2016;2(2):101-8.

29. Azadi A, Hamidi M, Rouini M-R. Methotrexateloaded chitosan nanogels as 'Trojan Horses' for drug delivery to brain: preparation and in vitro/in vivo characterization. Int $\mathrm{J}$ Biol Macromolec. 2013;62:523-30. doi: 10.1016 / j.ijbiomac .2013 .10 .004

30. Costa P, Lobo JMS. Modeling and comparison of dissolution profiles. Eur J Pharm Sci. 2001;13(2):123-33.

31. Shinichiro H, Takatsuka Y, Tai M, Hiroyuki M. Absorption of drugs from the nasal mucosa of rat. Int J Pharm. 1981;7(4):317-25. doi: 10.1016/03785173(81)90058-2.

32. Azadi A. Roini MR. Hamidi M. Neuropharmacokinetic evaluation of methotrexate loaded chitosan nanogels. Int J Biol Macromol. 2015; 79: 326-35. Doi: 10.1016/ j. ijbiomac.2015.05.001.

33. Ashrafi H, Azadi A. Chitosan-based hydrogel nanoparticle amazing behaviors during transmission electron microscopy. Int J Biol Macromol. 2016;84:31-4. doi: 10.1016/j.ijbiomac.2015.11.089.

34. Baldrick $P$. The safety of chitosan as a pharmaceutical excipient. Regulatory Toxicology and Pharmacology. 2010;56(3):290-9. doi: 10.1016/j.yrtph.2009.09.015.

35. Hamidi M. Role of P-glycoprotein in tissue uptake of indinavir in rat. Life Sci. 2006;79(10):991-8. doi: 10.1016/j.lfs.2006.05.010.

36. Mistry A, Stolnik S, Illum L. Nanoparticles for direct nose-to-brain delivery of drugs. Int $\mathrm{J}$ Pharm. 2009;379(1):146-57. doi: 10.1016 / j.ijpharm .2009.06.019.

37. Gabal YM, Kamel AO, Sammour OA, Elshafeey AH. Effect of surface charge on the brain delivery of nanostructured lipid carriers in situ gels via the nasal route. Int J Pharm. 2014;473(1):442-57. doi: 10.1016/j.ijpharm.2014.07.025.

38. Bitter C, Suter-Zimmermann K, Surber C. Nasal drug delivery in humans. Curr Probl Dermatol. 2011. doi: $10.1159 / 000321044$.

39. Bhattarai N, Gunn J, Zhang M. Chitosan-based hydrogels for controlled, localized drug delivery. Adv Drug Deliv Rev. 2010;62(1):83-99. doi: 10.1016/j.addr.2009.07.019.

40. Barzegar-Jalali M, Adibkia K, Valizadeh H, Shadbad MRS, Nokhodchi A, Omidi Y, et al. Kinetic analysis of drug release from nanoparticles. J Pharm Pharm Sci. 2008;11(1):167-77. 
41. Siepmann J, Siepmann F. Mathematical modeling of drug delivery. Int J Pharm. 2008;364(2):328-43. doi: 10.1016/j.ijpharm.2008.09.004.

42. Azadi S, Ashrafi H, Azadi A. Mathematical modeling of drug release from swellable polymeric nanoparticles. J Appl Pharm Sci. 2017; 7(04):125-33.

43. Shingaki $T$, Inoue $D$, Furubayashi $T$, Sakane $T$, Katsumi H, Yamamoto A, et al. Transnasal delivery of methotrexate to brain tumors in rats: a new strategy for brain tumor chemotherapy. Mol Pharm. 2010;7(5):1561-8. doi: 10.1021/mp900275s.

44. Wang F, Jiang X, Lu W. Profiles of methotrexate in blood and CSF following intranasal and intravenous administration to rats. Int J Pharm. 2003;263(1):1-7. 